\title{
Effect of Integrating Maternal and Child Health Services, Nutrition and Family Planning Services on Postpartum Family Planning Uptake at 6 Months Post-partum in Burkina Faso, Cote d'Ivoire and Niger: Protocol of a Longitudinal Quasi-experimental Study
}

DENISE KPEBO ( $\square$ dkpebo@gmail.com )

Université Félix Houphouët-Boigny UFR des Sciences Médicales: Universite Felix Houphouet-Boigny Unite de Formation et de Recherche des Sciences Medicales https://orcid.org/0000-0002-5185-2516

Abou Coulibaly

Institut de Recherche en Sciences de la Sante

Maurice Yameogo

Institut Africain de Santé Publique: Institut Africain de Sante Publique

Sujata Bijou

IntraHealth: IntraHealth International Inc

Lazoumar Ramatoulaye

CERMES: Centre de Recherche Medecine Sciences Sante Sante Mentale Societe

Halima Tougri

Institut de Recherche en Sciences de la Sante

Marguerite N'dour

IntraHealth: IntraHealth International Inc

Seni Kouanda

Institut de Recherche en Sciences de la Sante

\section{Study protocol}

Keywords: Maternal and infant health, Postpartum famly planning, Integration of services, Maternal-Child Health Services / organization \& administration

Posted Date: January 4th, 2022

DOI: https://doi.org/10.21203/rs.3.rs-1094966/v1

License: (c) (i) This work is licensed under a Creative Commons Attribution 4.0 International License. Read Full License 


\section{Abstract}

Background : Although several interventions integrating maternal, neonatal, child health and nutrition with family planning have been implemented and tested, there is still limited evidence on their effectiveness to guide program efforts and policy action,on health services integration. This study aims to assess the effectiveness of a service delivery model integrating maternal and child health services, nutrition and family planning services, compared with the general standard of care in Burkina Faso, Cote d'Ivoire, and Niger.

Methods: This is a quasi experimental study with one intervention group and one control group of 3-4 health facilities in each country. Each facility was matched to a control facility of the same level of care and that had similar coverage on selected reproductive health indicators such as family planning and post-partum family planning.

The study participants are pregnant women (with a 6 months pregnancy at maximum) coming for their first antenatal care visit. They will be followed up to 6 months after childbirth, and will be interviewed at each antenatal visit and also during visits for infant vaccines.

The analyzes will be carried out by intention to treat, using generalized linear models (binomial log or log Poisson) to assess the effect of the intervention on the ratio of contraceptive use prevalence between the two groups of the study at a significance level of $5 \%$, while taking into account the cluster effect and adjusting for potential confounding factors (socio-demographic characteristics of women, unevenly distributed at inclusion).

Discussion :This longitudinal study, with the provision of family planning services integrated into the whole maternal care continuum, a sufficiently long observation time and repeated measurements, will make it possible to better appreciate the timeline and the factors influencing women's decision-making on the use of post-partum family planning services. The results will help in increasing the body of knowledge regarding the impact of maternal and child health services integration on the utilization of post-partum family planning, taking into account the specific context of sub-Saharan Africa French speaking countries where such information is very needed.

\section{Plain English Summary}

One strategy to improve the utilization of health services by mothers and their children is the integration of maternal and child health services. For instance, a pregnant woman coming for an antenatal care visit would also receive counseling on post-partum family planning services and maternal nutrition. Similarly, a woman coming for her infant's vaccines would be offered counseling on post-partum family planning, maternal nutrition and breastfeeding.

Although several interventions have been implemented and tested, there is still limited evidence on the conditions and factors required for successful maternal and child health services integration strategies. This study aims to assess the effectiveness of an intervention integrating maternal and child health services, nutrition and family planning services.

For the purpose of the evaluation, 2 distincts groups of health facilities will be selected in each country, one group of 3-4 health facilities where the intervention will be implemented, and another group of 3-4 health facilities with the general standard of care. The study participants are pregnant women coming for their for their first antenatal care visit, who will be followed up to 6 months after childbirth. The analyzes will be carried out to assess the effect of the intervention on contraceptive use prevalence between the two groups of health facilities.

This study will make it possible to better appreciate the timeline and the factors influencing women's decision-making on the use of postpartum family planning services.

\section{Background}

In 2017, an estimated 295,000 women died from pregnancy-related causes, half of these maternal deaths occurred in the third trimester of pregnancy, and 2.6 million babies died at birth[1, 2]. The brunt of these deaths is particularly high in Sub-Saharan Africa (SSA) as this region account for approximately $66 \%$ of the estimated global maternal deaths in 2017 , and about $53 \%$ of all under-five deaths in 2019 , with a neonatal mortality rate that is twice as high as the rate at the global level $(76 \%$ vs $38 \%$ o $)[1,2]$. The situation is particularly alarming in the countries of the Ouagadougou partnership, 9 French speaking countries in western SSA. It is estimated that one in 41 women die from maternal causes in these countries, compared to 1 in 54 in developping countries, and 1 in 4,900 in developed countries. Neonatal mortality rates in the region vary from $22 \%$ o (Senegal) to $32 \%$ (Mali) [2].

Effective interventions to reduce maternal and newborn mortality are well known. Skilled care before, during, and after childbirth can reduce maternal morbidity and mortality [3]. Neonatal mortality can be reduced by skilled birth attendance at delivery, newborn resuscitation, 
exclusive breastfeeding, umbilical cord care and management of infections in newborns, and child immunization [4]. Also, births spacing and the use of effective contraceptive methods can improve maternal, perinatal, and neonatal health, by reducing stillbirths, prematurity, low birth weight and neonatal and maternal mortality $[5,6]$.

Although these interventions are known, their coverage rates remain low [6]. Recent data show that while most women (over $80 \%$ ) receive at least one antenatal care visit (ANC), only $49.3 \%$ receive 4 to 7 visits and $11.3 \%$ receive 8 visits [7]. The unmet need for family planning (FP) remains large and is concentrated in the postpartum period. It is estimated that this need, soon after birth, reaches $75 \%$ in West and Central Africa [8].

Given the importance of these interventions, delivering them as part of a comprehensive package could help ensure maximum benefits for the mother-child dyad. The importance of integrating maternal, neonatal, and child health and nutrition (MNCHN) with FP is well recognized as a key strategy, particularly for reducing maternal and child mortality [7, 9]. These two areas are integral to successfully achieving the 2030 Sustainable Development Goals for improving maternal, neonatal and child health $[7,9,10]$. In addition, evidence showed that integrating $\mathrm{MNCH}$ and FP services would cost approximately $\$ 1.5$ billion less than providing $\mathrm{MNCH}$ services alone [11].

Although, several integrated service delivery initiatives targeting mother and child during pregnancy and early childhood have been implemented and tested [12-16], there is still limited information and evidence to guide policy action, program efforts, and scaling-up strategies on health services integration. Indeed, most of these interventions presented shortcomings not only at the conceptual level but also in terms of the methodology used[17]. In general, these integration initiatives rarely involved more than two services, and nutriton services were generally poorly included among the services offered [18-20]. Then, the evaluations of the effects of these interventions focused mainly on the coverage of services $[11,14,17]$. Finally, the evaluation design used are not always robust; most of them being mere before and after study design without any control group $[13,21]$.

In order to fill these gaps, we have developed this protocol for a longitudinal quasi experimental study. The general objective of this study is to assess the effectiveness of a service delivery model integrating maternal and child health services, nutrition and family planning services, compared with the general standard of care in Burkina Faso, Cote d'Ivoire, and Niger. Specifically, this study aims at (i) assessing the effects of the intervention on the uptake of post-partum family planning (PPFP) at 6 months post-partum, (ii) assessing the effects of the intervention on the use of integrated health services, (iii) and assessing the effects of the intervention on improving maternal and neonatal health indicators during pregnancy and the immediate postpartum period.

\section{Methods And Study Design Description of the intervention}

The model of integrated health service delivery includes the community level (community contacts with the community health workers), the intermediate level (rural and urban basic health centers), and the central level with the district hospital. The standard model of integrated PPFP / MNCH / Nutrition service delivery has four points of contact where integrated services should be offered in the health facility: antenatal care, childbirth, postnatal care, and infant wellness visits (Figure 1). In the model, these services are delivered during the same client visit based on client needs and standards of care.

\section{Study settings}

The study will be implemented in three French speaking countries of sub-Saharan West Africa: Burkina Faso, Cote d'Ivoire, and Niger. Some information related to relevant reproductive health and health service utilization indicators based on demographic and health survey data from Burkina Faso (2010), Cote d'Ivoire (2011-2012) and Niger (2012) are presented in Table 1.

Table 1

Selected indicators on reproductive health and utilization of maternal and child health services Burkina Faso (2010), Cote d'Ivoire (20112012) and Niger (2012) 


\begin{tabular}{|llll|}
\hline Selected indicators & Burkina Faso & Cote d'Ivoire & Niger \\
\hline Maternal mortality rate & $\begin{array}{l}330 \text { per 100, 000 live } \\
\text { births in 2015 }\end{array}$ & $\begin{array}{l}614 \text { per100, 000 live } \\
\text { births in 2015 }\end{array}$ & $\begin{array}{c}520 \text { pour 100, 000 live } \\
\text { births in 2015 }\end{array}$ \\
\hline Neonatal Mortality Rate & 23 per1,000 & 33 per 1,000 & 28 per 1,000 \\
\hline Child Mortality Rate & 42 per 1,000 & 27 per 1,000 & 48 per 1,000 \\
\hline mCPR & $22.5 \%$ & $23.5 \%$ & $11 \% *$ \\
\hline Unmet need of FP & $23.3 \%$ & $39.2 \%$ & $15 \%^{*}$ \\
\hline Prevalence of acute malnutrition & $8.6 \%$ & $6 \%$ & $10.3 \%$ \\
\hline Prevalence of chronic malnutrition & $21.2 \%$ & $21.6 \%$ & $42.2 \%$ \\
\hline Prevalence of underweight & $16.2 \%$ & $12.8 \%$ & - \\
\hline Early breastfeeding & $56 \%$ & $37 \%$ & $30 \%$ \\
\hline $\begin{array}{l}\text { Proportion of infants under } 6 \text { months of age who have } \\
\text { benefited from Exclusive Breastfeeding }\end{array}$ & $47.8 \%$. & $23.5 \%$ & $55.6 \%$ \\
\hline Proportion of anemia in pregnant women & $58 \%$ & $64 \%$ & \\
\hline$*$ Among women in union & & & \\
\hline
\end{tabular}

\section{Type of study}

This is a quasi experimental study; in each country, we will have one intervention group of facilities and one control group of facilities.

Participants of the intervention group, meaning women attending the intervention facilities, will receive the full package of integrated PPFP / $\mathrm{MNCH} /$ Nutrition services, while participants in the control group will receive standard care.

\section{Sites of study}

In each country, 3 to 4 health facilities have been identified as the intervention sites including a district hospital, an urban health center, and a rural health center. Each facility was matched to a control facility of the same level of care and that had similar coverage on selected reproductive health indicators such as family planning and post-partum family planning.

Table 2

Sites of study

\begin{tabular}{|c|c|c|}
\hline Country & Intervention settings & Control settings \\
\hline \multirow[t]{3}{*}{ Burkina Faso } & - CMA of Po & - CMA of Kombissiri \\
\hline & - CSPS niché at the CMA & - CSPS niche at the CMA \\
\hline & - CSPS of Tiébélé & - CSPS de Toécé \\
\hline \multirow[t]{3}{*}{ Cote d'Ivoire } & - HG district of Agnibilekro & - HG of Adzopé \\
\hline & - CSU of Damé & - CSU Assikoi \\
\hline & - CSR of Assuamé & - CSR Ananguié \\
\hline \multirow[t]{4}{*}{ Niger } & - HD of Aguié & - HD Guidanroumji \\
\hline & - CSI urban of Aguié & - CSU urban of Guidanroumji \\
\hline & - CSI rural of Débi & - CSI rural of Karazome \\
\hline & - CS of Zabon Moussou & - CS of Tabouka \\
\hline
\end{tabular}

HD, CMA, and HG= General Hospital or Health District Hospital // CSPS, CSU, CSR, CSI= Primary Health care facilities (CSU= urban, CSR=rural) 


\section{Study participants}

The study participants are pregnant women who meet the following criteria :

- Attending maternal health services for ANC;

- Having a 6 months pregnancy at maximum;

- Being a resident of the communities served by the health facility;

- Not planning to travel for more than one month during pregnancy or for six months after childbirth;

- Having the intention from the outset to follow preventive care and childbirth in the health facility.

\section{Participants recruitment process}

Participants will be recruited at the ANC unit. All women coming for their first ANC visit will be invited to participate in the study. Women who accept will be screened according to the inclusion criteria. Those meeting the study criteria will be included in the study. In addition, each woman will have a unique identification number which will be entered in the registers to allow follow up, throughout the duration of the study.

\section{Participant follow-up procedures}

Women (with a 6 months pregnancy at maximum) will be followed up to 6 months after childbirth, and will be interviewed at each antenatal visit and also during visits for infant vaccination, as detailed in the table 3 below.

Table 3

Participants follow-up schedule

\begin{tabular}{|c|c|c|c|c|c|c|c|c|c|c|c|c|c|c|c|}
\hline & ANC1 & ANC2 & ANC3 & ANC4 & ANC5 & ANC6 & ANC7 & ANC8 & $\begin{array}{l}\text { Deli- } \\
\text { very }\end{array}$ & $\begin{array}{l}\text { Early } \\
\text { PNC } \\
\text { Day6- } \\
10\end{array}$ & $\begin{array}{l}\text { Late } \\
\text { PNC } \\
\text { Day42 } \\
-56\end{array}$ & M2 & M3 & M4 & M6 \\
\hline Admission & $X$ & & & & & & & & & & & & & & \\
\hline Follow-up & & $X$ & $X$ & $X$ & $X$ & $X$ & $X$ & $X$ & $X$ & $X$ & $X$ & $X$ & $X$ & $X$ & $X$ \\
\hline
\end{tabular}

\section{Outcomes measures}

Case-report forms (CRFs) will be developed for collecting data on the outcomes of interest. The primary outcome is the uptake of modern contraceptive methods at six months postpartum (proportion of women using modern contraceptives at six months in the experimental vs. control group). Secondary outcomes are related to health indicators and services utilization, as detailed in the table 4 below. Maternal infections will be considered for clinical signs of postpartum infection in mothers before discharge from hospital. Early neonatal infections will be considered as reported by health care providers in the patient file or based on obvious clinical signs of neonatal infection (fever, hypothermia, jaundice)

Table 4

Secondary outcomes of interest to be measured 


\begin{tabular}{|c|c|c|}
\hline \multicolumn{3}{|l|}{ Secondary Outcomes of interest } \\
\hline Health indicators & Services delivered & Services utilization \\
\hline Early breastfeeding & $\begin{array}{l}\text { Number of individual FP counseling sessions during } \\
\text { ANC }\end{array}$ & Number of ANC visits (retention) \\
\hline Birth weight & Nutritional advice received during ANC & Number of Post-partum visits \\
\hline Exclusive breastfeeding 0-6 months & Nutritional advice received during post- natala care & $\begin{array}{l}\text { Infant growth monitoring } \\
\text { (weighing) }\end{array}$ \\
\hline \multicolumn{3}{|l|}{ Moderate acute malnutrition } \\
\hline \multicolumn{3}{|l|}{ Severe acute malnutrition } \\
\hline \multicolumn{3}{|l|}{$\begin{array}{l}\text { Vaccine coverage for children } 0-6 \\
\text { months }\end{array}$} \\
\hline \multicolumn{3}{|l|}{$\begin{array}{l}\text { Vitamin A supplementation and } \\
\text { deworming }\end{array}$} \\
\hline \multicolumn{3}{|l|}{ Cough } \\
\hline \multicolumn{3}{|l|}{ Diarrhea } \\
\hline \multicolumn{3}{|l|}{ Malaria } \\
\hline \multicolumn{3}{|l|}{ Maternal infections } \\
\hline Neonatal infections & & \\
\hline
\end{tabular}

\section{Sample size calculation}

According to the Population Division of the United Nations Department of Economic and Social Affairs, the modern contraceptive prevalence among married or in union women in 2020 was estimated at 22.4\% Cote d'Ivoire, 28.1\% for Burkina Faso and 15.1\% for Niger [24].

Considering an improvement of $15 \%$ in this proportion, attributable to the intervention, with a power of $80 \%$, a significance level of $5 \%$, and finally an intraclass correlation coefficient of 0.015 , Cote d'Ivoire will have the largest sample size, about 88 women per cluster. By increasing the size by $15 \%$ to take into account any lost to follow-up related to travel, we'll have a size of 102 women per health facility, meaning a total of 306 women per group in Cote d'Ivoire, and 268, and 172 per group in Burkina Faso and Niger, respectively. Details for each country are provided in the table 5 below.

Table 5

Parameters used for sample size calculation 


\begin{tabular}{|llll|}
\hline Caracteristics & Cote d'ivoire & Burkina Faso & Niger \\
\hline Power & $80 \%$ & $80 \%$ & $80 \%$ \\
\hline modern Contraceptive Prevalence rate & $22.4 \%$ & $28.1 \%$ & $15.1 \%$ \\
\hline Size of the expected effect in intervention groups & $15.0 \%$ & $15.0 \%$ & $15.0 \%$ \\
\hline modern Contraceptive Prevalence rate (expected) & $37.4 \%$ & $43.1 \%$ & $30.1 \%$ \\
\hline Power & $80 \%$ & $80 \%$ & $80 \%$ \\
\hline Significance & $5 \%$ & $5 \%$ & $5 \%$ \\
\hline Intercluster Correlation Coefficient & 0.015 & 0.015 & 0.015 \\
\hline Size per cluster & 88 & 58 & 37 \\
\hline Size increased per cluster (15\% increase) & 102 & 67 & 43 \\
\hline Number of clusters per group & 3 & 4 & 4 \\
\hline Final size of groups & 306 & 268 & 172 \\
\hline Final Size by country & 612 & 536 & 344 \\
\hline
\end{tabular}

\section{Data collection}

Data will be collected through direct interview with health facility clients and extraction of data from health facility registers.

Direct interview with health facility clients: They will be carried out with the study participants at the various follow-up points. Data will be collected from a standardized questionnaire integrated into electronic tablets. Interviews will be carried out in health centers on the day of the woman's consultation at her convenience. Otherwise, the interviewer will get in touch with the participant to agree on a day and place for the interview. In addition, these interviews will comply to barrier measures against Covid-19 (wearing a mask and a maintaining a distance of at least one meter between the investigator and the respondent)

Data extraction: Data will be extracted from health center registers (ANC, Childbirth, post-natal care, Immunization).

\section{Recruitment and training of investigators}

Data will be collected by 06 females interviewers per country, with a paramedical profile (nurse, midwife), speaking the local languages.

They will be trained for one week on the study procedures and the content of the questionnaire.

\section{Data quality procedures}

Data quality control procedures will be put in place to ensure that accurate data are recorded in the registers and entered into the database. Guidelines for data collection and the establishment of a registration register will be produced. In order to ensure that data will be collected in a standardized way in all participating health facilities, a pilot test of data collection and data management will be carried out before the beginning of baseline data collection. Data quality reports will be produced regularly for each health facility. Site control supervisions in the participating health facilities will be carried out regularly and a verification of the source data will be carried out to ensure that the data collected is accurate, complete, , precise and reliable.

The supervisions will be carried out by the principal investigator and the country co-investigators.

\section{Data analysis}

A statistical analysis plan will be developed. Descriptive statistics will be reported by calculating frequencies and percentages for categorical variables and means, standard deviations, and minimum and maximum values for the continuous variables. The distribution of variables will 
be examined for outliers as part of quality control and descriptive analysis of the data. Descriptive statistics will be compiled for each group and aggregated between groups.

The analysis will compare the unadjusted and adjusted primary results between the two study groups, i.e. the experimental group and the comparison group. Since the main outcome is contraceptive use, we will use generalized linear models (binomial log or log Poisson) to assess the effect of interventions on the ratio of contraceptive use prevalence between the two groups of the study at a significance level of $5 \%$, while taking into account the cluster effect and adjusting for potential confounding factors (socio-demographic characteristics of women, unevenly distributed at inclusion). All bi and multivariate analyzes will be carried out by intention to treat, including all women, whether or not they continued to visit health centers after inclusion. The unit of analysis will therefore be the woman. The analyzes will be carried out with the Stata software.

\section{Ethics}

This protocol has been approved by the Institutional Review Committee of Intrahealth International as well as the respective ethical committees of the selected countries.

To ensure the safety and well-being of participants (healthcare providers and pregnant women) and to ensure no harm to them for this study, the team will take the following measures:

- All women meeting the inclusion criteria will be provided with detailed information on the objectives and procedures of the study and free and informed consent will be required prior to inclusion.

o Pregnant women under 18 who are in union will be considered as emancipated minors and consent will be obtained directly as for an adult.

o For pregnant women under the age of 18 who are not in a union, the consent of an adult parent / legal guardian will be required as well as the consent of the young girl.

- All research investigators and study staff will be trained to clearly communicate and perform the consent process.

There will be no risk to women who decide not to participate in the study. Women who will not consent to participate in the study will receive the same care and access to services as those who have consented to participate in the study. If a condition warranting referral (including domestic violence, substance abuse, etc., HIV counseling and testing, or any other relevant condition) is detected during the provision of antenatal care services (as part of this research), the study team will ensure that the woman is correctly referred and that the appropriate standard referral procedures are followed.

- The data collected on the tablets will be sent directly to the local IRSS-based server once a week. The server will be protected by a password known only from the Data manager. All research assistants and study staff will be trained to ensure data security.

The published data will be depersonalized, described in a comprehensive manner, if possible and the anonymity of the participants will be preserved at all times.

\section{Study timeline}

This study will last 18 months, from July 2021 to December 2022 as follows:

- 4 months for participants recruitment;

- 11 months of follow-up from the last woman included (she will be recruited during the first trimester of pregnancy at earliest, therefore 56 months of follow-up while she is still pregnant and 6 months of follow-up after delivery);

- 3 months for the report writing.

\section{Discussion}

Although significant interest in integrating family planning with other health services emerged during the last 30 years, both for programmatic and political reasons, limited empirical evidence is available on the effectiveness of programs that integrate family planning with maternal, perinatal, and child health [14]. Moreover, there is a paucity of evidence from developing countries in terms of what intervention programs work best for PPFP $[11,14,25]$. Of the relatively very few studies on integration that have been conducted, most were limited by 
methodological quality including cross-sectional design, hospital based surveys, non-family planning outcomes as main interest, and also a short duration of observation $[25,26]$. Overall, it is recognized that the evidence of the integration of postpartum family planning with other health services remains weak, and well-designed evaluation research is needed [11, 14, 25-27].

Previous studies showed that the results of the implact of integration of PPFP services into maternal health services on uptake of contraceptive methods are mixed. Some studies have found a relationship between ANC and contraceptive adoption in the post-partum but information was missing on whether or not PPFP counseling was provided during the ANC sessions [28, 29]. Other studies did not find any relationship between the integration of PPFP counseling into ANC and the uptake of contraceptive method in the postpartum period, but did rather find an impact of these services integration on women's intention to use contraceptives methods [30]. However, integration of PPFP counseling into delivery care and postnatal care has shown more consistent positive impact on increasing adoption of PPFP [31-33].

As compared to these previous studies, one of the advantages of this intervention is that the provision of PPFP services will not be limited to just a few points of contact, but will rather be integrated into the whole maternal care continuum. In addition, this longitudinal study, with a sufficiently long observation time and repeated measurements, will make it possible to better appreciate the timeline and the factors influencing women's decision-making on the use of PPFP.

As for any multisite study, expected limitations and challenges regarding this study include coordination issues with and between study countries and study sites, political and policy changes, eventual constraints in delivering the intervention with fidelity, and maintaining the intervention timelines [34]. To mitigate these threats, a technical working group is set up in each country to follow the overall implementation of the intervention, including the evaluation study. This technical working group is comprised of several stakeholders including those of the $\mathrm{MOH}$ to ensure the country engagement in the study process. In addition, a study coordination team is set up in each country to facilitate coordination between the different countries and study settings. Finally, a journal of events will be held throughout the study implementation to monitor any factor or event that could influence study outcomes, so as to be considered during results analysis.

Our study, also has a number of strengths, primarily the longitudinal design will allow us to assess exposure to postpartum counseling and uptake of contraception at frequent intervals throughout the postpartum period, limiting recall bias. As we will collect information on ongoing pregnancy and health services utilization, we will also limit confounding due to temporal changes that are present when including past births. We therefore believe that the overall strengths of the proposed design outweigh its limitations and the coming results will help in increasing the body of knowledge on this topic, especially for SSA french speaking countries.

\section{Abbreviations}

\begin{tabular}{ll} 
ANC & Antenatal care visit \\
\hline CMA & Centre Médical avec antenne chirurgicale (Medical center with a surgical unit) \\
\hline CS & Case de santé (health hut) \\
\hline CSI urbain & Centre de Santé Intégré urbain (primary health care facility in urban area) \\
\hline CSI rural & Centre de Santé Intégré rural (primary health care facility in rural area) \\
\hline CSPS & Centre de santé et de promotion sociale (health and social promotion center) \\
\hline CSU & Centre de santé urban (primary health care facility in urban area) \\
\hline CSR & Centre de santé rural (primary health care facility in rural area) \\
\hline FP & Family planning \\
\hline HD & Hôpital de District (District level hospital) \\
\hline HG & Hôpital General (General hospital or district level hospital) \\
\hline MNCH & Maternal, neonatal, and child health services \\
\hline MNCHN & Maternal, neonatal, child health services and nutrition \\
\hline PPFP & Post-partum family planning \\
\hline SSA & sub-Sahara Africa
\end{tabular}

\section{Declarations}




\section{Ethics approval}

This protocol has been approved by the Institutional Review Committee of Intrahealth International under the log number 21004 . It has also been approved by the respective ethical committees of the selected countries as detailled below:

- Cote d'Ivoire, approval N N/Ref: 053-21/MSHP/CNESVS-km;

- Burkina Faso, approval N²021-000131/MS/MESRS/CERS;

- Niger, approval $N^{\circ} 53 / 2020 / C N E R S$

\section{Competing interest}

The author(s) declare(s) that they have no competing interests.

\section{Funding}

This study is part of a bigger project implemented by Intrahealth and funded by the Bill and Melinda Gates Foundation. For the implementation of the project described in this article, Intrahealth has issued a contract to IRSS, in Burkina Faso under the subgrant $\mathrm{n}^{\circ} 5037$ 001. However, no funding was available for this article.

\section{Consent for publication}

Not applicable, no individual data was used at this stage of the study.

\section{Availability of data and material}

Not applicable, no data or material was used at this stage of the study.

\section{Authors'contributions}

DK, AC and SK conceptualized the study project and led the protocol, and led the protocol writing process. MY, LR, and HT reviewed and adapted the study (including the protocol and tool) to each of their country settings. SB and MN reviewed the protocol for editing.All authors read and approved the final manuscript.

\section{Acknowledgements}

The authors acknowledged Intrahealth IntraHealth and the INSPIRE Project for implementing this project. The project was funded by the Bill and Melina Gates Foundation. We wish to acknowledge helpful key people in the study countries that were kind enough to provide additional information and explanations when needed.

\section{References}

1. Trends in Maternal Mortality. 2000 to 2017 [Internet]. [cited 2021 Nov 13]. Available from: https://www.unfpa.org/fr/node/20758.

2. Levels and trends in. child mortality 2020 | UNICEF [Internet]. [cited 2021 Nov 13]. Available from: https://www.unicef.org/reports/levelsand-trends-child-mortality-report-2020.

3. Nyfløt L, Sitras V. Strategies to reduce global maternal mortality. Acta Obstet Gynecol Scand. 2018 Jun;97(6):639-40.

4. Khan AA, Zahidie A, Rabbani F. Interventions to reduce neonatal mortality from neonatal tetanus in low and middle income countries - a systematic review. BMC Public Health. 2013 Apr;9(1):322. 13(.

5. Conde-Agudelo A, Rosas-Bermúdez A, Kafury-Goeta AC. Birth spacing and risk of adverse perinatal outcomes: a meta-analysis. JAMA. 2006 Apr;19(15):1809-23. 295(.

6. Conde-Agudelo A, Rosas-Bermúdez A, Kafury-Goeta AC. Effects of birth spacing on maternal health: a systematic review. Am J Obstet Gynecol. 2007 Apr;196(4):297-308.

Page $10 / 12$ 
7. Koblinsky M, Moyer CA, Calvert C, Campbell J, Campbell OMR, Feigl AB, et al. Quality maternity care for every woman, everywhere: a call to action. Lancet Lond Engl. 2016 Nov 5;388(10057):2307-20.

8. Rossier C, Bradley SEK, Ross J, Winfrey W. Reassessing Unmet Need for Family Planning in the Postpartum Period. Stud Fam Plann. 2015 Dec;46(4):355-67.

9. Black RE, Walker N, Laxminarayan R, Temmerman M. Reproductive. Maternal, Newborn, and Child Health: Key Messages of This Volume [Internet]. Reproductive, Maternal, Newborn, and Child Health: Disease Control Priorities, Third Edition (Volume 2). The International Bank for Reconstruction and Development / The World Bank; 2016 [cited 2021 Nov 13]. Available from: https://www.ncbi.nlm.nih.gov/books/NBK361926/.

10. Kuruvilla S, Bustreo F, Kuo T, Mishra C, Taylor K, Fogstad H, et al. The Global strategy for women's, children's and adolescents' health (2016-2030): a roadmap based on evidence and country experience. Bull World Health Organ. 2016 May;94(5)(1):398-400.

11. Cleland J, Shah IH, Daniele M. Interventions to Improve Postpartum Family Planning in Low- and Middle-Income Countries: Program Implications and Research Priorities. Stud Fam Plann. 2015 Dec;46(4):423-41.

12. Sitrin D, Jima GH, Pfitzer A, Wondimu C, Belete TW, Pleah T, et al. Effect of integrating postpartum family planning into the health extension program in Ethiopia on postpartum adoption of modern contraception. J Glob Health Rep. 2020 Aug 2;4:e2020058.

13. Yugbaré Belemsaga D, Goujon A, Tougri H, Coulibaly A, Degomme O, Duysburgh E, et al. Integration of maternal postpartum services in maternal and child health services in Kaya health district (Burkina Faso): an intervention time trend analysis. BMC Health Serv Res. 2018 Apr 23;18(1):298.

14. Baqui AH, Ahmed S, Begum N, Khanam R, Mohan D, Harrison M, et al. Impact of integrating a postpartum family planning program into a community-based maternal and newborn health program on birth spacing and preterm birth in rural Bangladesh. J Glob Health. 2018 Dec;8(2):020406.

15. Mackenzie D, Pfitzer A, Maly C, Waka C, Singh G, Sanyal A. Postpartum family planning integration with maternal, newborn and child health services: a cross-sectional analysis of client flow patterns in India and Kenya. BMJ Open [Internet]. 2018 Apr 3 [cited 2021 May 14];8(4). Available from: https://www.ncbi.nlm.nih.gov/pmc/articles/PMC5892750/.

16. Cooper CM, Ogutu A, Matiri E, Tappis H, Mackenzie D, Pfitzer A, et al. Maximizing Opportunities: Family Planning and Maternal, Infant, and Young Child Nutrition Integration in Bondo Sub-County, Kenya. Matern Child Health J. 2017;21(10):1880-9.

17. Dulli LS, Eichleay M, Rademacher K, Sortijas S, Nsengiyumva T. Meeting Postpartum Women's Family Planning Needs Through Integrated Family Planning and Immunization Services: Results of a Cluster-Randomized Controlled Trial in Rwanda. Glob Health Sci Pract. 2016 Mar 25;4(1):73-86.

18. Zimmerman LA, Yi Y, Yihdego M, Abrha S, Shiferaw S, Seme A, et al. Effect of integrating maternal health services and family planning services on postpartum family planning behavior in Ethiopia: results from a longitudinal survey. BMC Public Health. 2019 Nov 4;19(1):1448.

19. Achyut P, Mishra A, Montana L, Sengupta R, Calhoun LM, Nanda P. Integration of family planning with maternal health services: an opportunity to increase postpartum modern contraceptive use in urban Uttar Pradesh, India. J Fam Plann Reprod Health Care. 2016 Apr 1;42(2):107-15.

20. Nguyen PH, Kim SS, Sanghvi T, Mahmud Z, Tran LM, Shabnam S, et al. Integrating Nutrition Interventions into an Existing Maternal, Neonatal, and Child Health Program Increased Maternal Dietary Diversity, Micronutrient Intake, and Exclusive Breastfeeding Practices in Bangladesh: Results of a Cluster-Randomized Program Evaluation. J Nutr. 2017 Dec;147(12):2326-37.

21. Yugbaré Belemsaga D, Goujon A, Bado A, Kouanda S, Duysburgh E, Temmerman M, et al. Integration of postpartum care into child health and immunization services in Burkina Faso: findings from a cross-sectional study. Reprod Health. 2018 Oct;11(1):171. 15(.

22. Yugbaré Belemsaga D, Goujon A, Tougri H, Coulibaly A, Degomme O, Duysburgh E, et al. Integration of maternal postpartum services in maternal and child health services in Kaya health district (Burkina Faso): an intervention time trend analysis. BMC Health Serv Res [Internet]. 2018 Dec [cited 2018 Jul 10];18(1). Available from: https://bmchealthservres.biomedcentral.com/articles/10.1186/s12913018-3098-6.

23. Yugbaré Belemsaga D, Bado A, Goujon A, Duysburgh E, Degomme O, Kouanda S, et al. A cross-sectional mixed study of the opportunity to improve maternal postpartum care in reproductive, maternal, newborn, and child health services in the Kaya health district of Burkina Faso. Int J Gynecol Obstet. 2016 Nov;135(S1):20-6.

24. United Nations Department of Economic and Social Affairs, Population Division. (2020). United Nations Department of Economic and Social Affairs, Population Division (2020). World Fertility and Family Planning 2020: Highlights (ST/ESA/SER.A/440).

25. Bain-Brickley D, Chibber K, Spaulding A, Azman H, Lindegren ML, Kennedy CE, et al. Strategies for integrating family planning services with maternal, neonatal and child health, and nutrition services. Cochrane Database Syst Rev [Internet]. 2011 [cited 2021 Nov 17];(7). Available from: https://www.cochranelibrary.com/cdsr/doi/10.1002/14651858.CD009222/full.

Page 11/12 
26. Final C. Report 81611 clean.pdf [Internet]. [cited 2021 May 14]. Available from:

https://toolkits.knowledgesuccess.org/sites/default/files/Final\%20Comprehensive\%20Report\%208\%2016\%2011\%20clean.pdf.

27. Lindegren ML, Kennedy CE, Bain-Brickley D, Azman H, Creanga AA, Butler LM, et al. Integration of HIV/AIDS services with maternal, neonatal and child health, nutrition, and family planning services. Cochrane Database Syst Rev. 2012 Sep 12;(9):CD010119.

28. Do M, Hotchkiss D. Relationships between antenatal and postnatal care and post-partum modern contraceptive use: evidence from population surveys in Kenya and Zambia. BMC Health Serv Res. 2013 Jan;4(1):6. 13(.

29. Zerai A, Tsui AO. The relationship between prenatal care and subsequent modern contraceptive use in Bolivia, Egypt and Thailand. Afr J Reprod Health. 2001 Aug;5(2):68-82.

30. Keogh SC, Urassa M, Kumogola Y, Kalongoji S, Kimaro D, Zaba B. Postpartum Contraception in Northern Tanzania: Patterns of Use, Relationship to Antenatal Intentions, and Impact of Antenatal Counseling. Stud Fam Plann. 2015 Dec;46(4):405-22.

31. Speizer IS, Fotso JC, Okigbo C, Faye CM, Seck C. Influence of integrated services on postpartum family planning use: a cross-sectional survey from urban Senegal. BMC Public Health. 2013 Aug;14:13:752.

32. Hounton S, Winfrey W, Barros AJD, Askew I. Patterns and trends of postpartum family planning in Ethiopia, Malawi, and Nigeria: evidence of missed opportunities for integration. Glob Health Action. 2015;8:29738.

33. Blazer C, Prata N. Postpartum family planning: current evidence on successful interventions. Open Access J Contracept. $2016 ; 7: 53-67$.

34. Tran NT, Gaffield ME, Seuc A, Landoulsi S, Yamaego WME, Cuzin-Kihl A, et al. Effectiveness of a package of postpartum family planning interventions on the uptake of contraceptive methods until twelve months postpartum in Burkina Faso and the Democratic Republic of Congo: the YAM DAABO study protocol. BMC Health Serv Res [Internet]. 2018 Jun 11 [cited 2021 Apr 11];18. Available from: https://www.ncbi.nlm.nih.gov/pmc/articles/PMC5996535/.

\section{Figures}

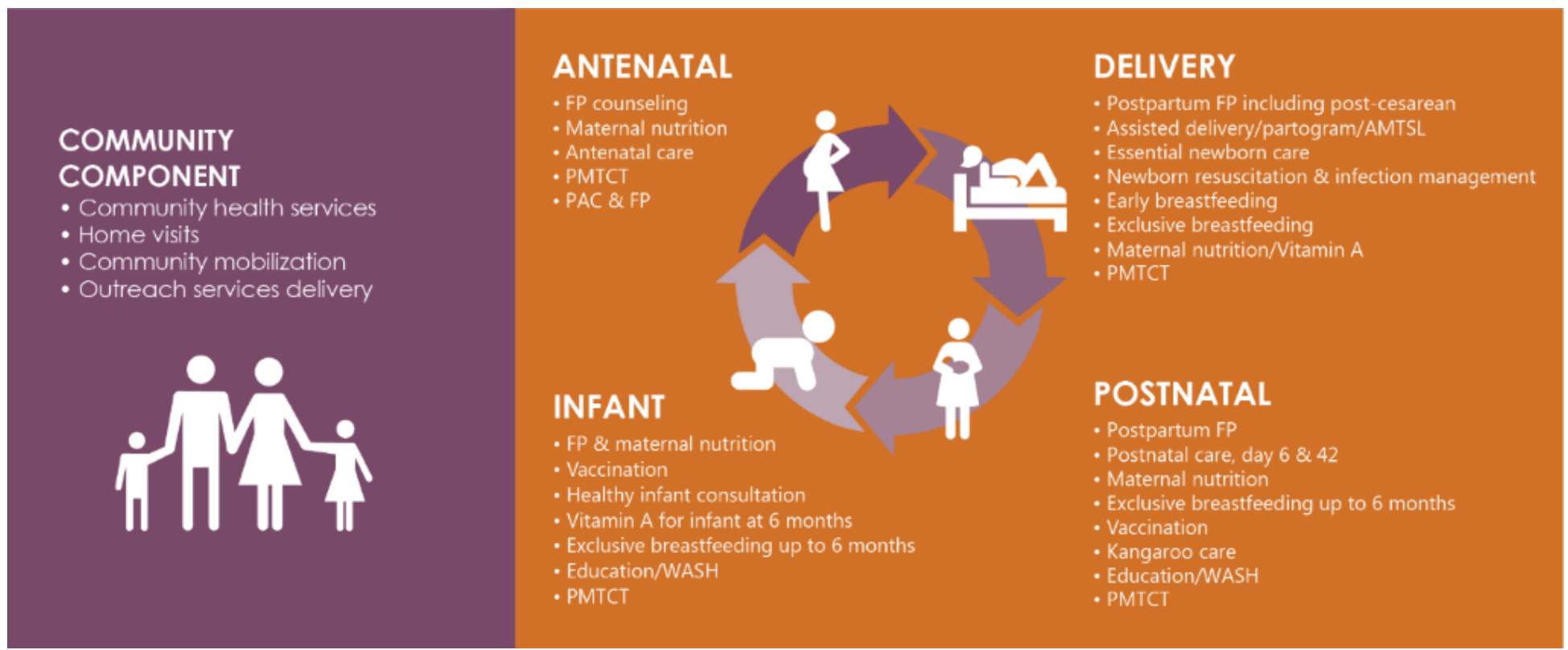

\section{Figure 1}

The intervention of integrated health services delivery 